\section{INCIDENTAL EXTRA-CARDIAC FINDINGS ON CLINICAL CMR; A COMPARISON OF 3 HASTE TECHNIQUES}

doi:10.1136/heartjnl-2011-300198.121

${ }^{1} \mathrm{R}$ B Irwin, ${ }^{2} \mathrm{~T}$ Newton, ${ }^{3} \mathrm{C}$ Peebles, ${ }^{4} \mathrm{~A}$ Borg, ${ }^{5} \mathrm{D}$ Clark, ${ }^{4} \mathrm{C}$ Miller, ${ }^{6} \mathrm{~N}$ Abidin, ${ }^{4} \mathrm{M}$ Greaves, ${ }^{4} \mathrm{M}$ Schmitt. 'Wythenshawe Hospital, Manchester, UK; ${ }^{2}$ Royal Blackburn Infirmary, Blackburn, UK; ${ }^{3}$ Southampton General Hospital, Southampton, UK; ${ }^{4}$ Wythenshawe Hospital, University Hospitals of South Manchester NHS Trust, Manchester, UK; ${ }^{5}$ Alliance Medical, Wythenshawe Hospital CME unit, Manchester, UK; ${ }^{6}$ Salford Royal Hospital, Salford, UK

Introduction Cardiac magnetic resonance (CMR) is an increasingly important imaging modality, which by necessity incorporates a large field of view. Both "localiser" and multiple slice half-fourier spin echo (eg, HASTE) sequences provide coverage of the thorax and upper abdomen. Such imaging may reveal hitherto unexpected incidental extra-cardiac findings (IEF). First we sought to assess the frequency of IEF found on clinically indicated CMR scans. Second we compared the 3 clinically used HASTE acquisition protocols in this context. Lastly we determined the impact of the 3 different protocols on acquisition time and image quality.

Methods Three subsequent groups of 238 patients (714 patients in total), all referred for clinically indicated CMR, were scanned with either breath-hold (BH) HASTE (Group 1), free breathing (FB) HASTE (Group 2) or diaphragmatic navigated (NAV) HASTE (Group 3). Additionally "localiser" sequences performed in 3 orthogonal planes were analysed. All 714 clinical reports were reviewed regarding the presence of IEF. These were categorised as either minor, or major if recommendations for further investigation, follow-up, and/or clinical correlation were made. Finally, to determine the impact of each HASTE protocol on acquisition time and image quality, an additional cohort of 15 patients underwent 3 protocols back to back in a random fashion. The length of each acquisition was timed and image quality was reviewed and scored externally.

Results A total of 180 IEF were found in 162 (22.7\%) out of 714 patients. There was no significant difference in frequency of IEF between the 3 HASTE groups. Out of 180 IEF, 88 were considered minor and 92 major findings. Of the latter, 8 (1.1\%) were considered highly significant. These included one bronchoalveolar carcinoma stage $1 \mathrm{~B}$ requiring lobectomy, 2 cases of florid sarcoidosis in patients presenting with VT and "structurally normal hearts" on echocardiography, one case of pulmonary aspergillosis, 2 cases of advanced pulmonary fibrosis, one ascending thoracic aortic aneurysm and a case of iatrogenic liver haemorrhage following placement of a pericardial drain. FB HASTE acquisition $(69 \pm 2.5 \mathrm{~s})$

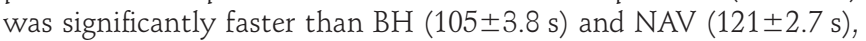
$\mathrm{p}<0.001$, but also produced the lowest image quality on a 5 point scale; $3.5(\mathrm{FB})$ vs $3.9(\mathrm{BH})$ vs $3.8(\mathrm{NAV}), \mathrm{p}=0.08$.

Conclusion Overall, IEF are common and lead to follow on investigations in a substantial minority of cases. However, the overall incidence of highly significant findings in the current study was low ( $\sim 1 \%)$. There was no difference in the frequency of incidental extracardiac findings between the 3 HASTE protocols. While the free breathing HASTE technique is statistically significantly faster than breath hold and navigated HASTE, the absolute time saving is small and probably out-weighted by lesser image quality.

\section{2}

OBESITY AND PERIVASCULAR ADIPOSITY IN ATHEROSCLEROSIS

doi:10.1136/heartjnl-2011-300198.122

I Kylintireas, C Shirodaria, 0 Rider, J M Lee, I Bechar, J Digby, M D Robson, $S$ Neubuer, R P Choudhury. University of Oxford, Oxford, UK

Introduction It has been proposed that perivascular adipose tissue (PVAT) contributes to inflammation and advancement of athero- sclerosis via a direct paracrine or vasocrine route. Excess adipose tissue accumulation leads to adipose tissue dysfunction characterised by a pro-inflammatory and potentially pro-atherogenic pattern of adipokine secretion. We used MRI for PVAT imaging and quantification and evaluated the effects of obesity and increased perivascular adiposity on the relationship of PVAT with the function and structure of the underlying vessels.

Methods We measured peri-aortic fat, aortic stiffness and atheroma burden by MRI in 128 cardiovascular patients and in 18 healthy lean subjects at baseline and in 22 healthy obese subjects (before and after weight loss intervention (diet or bariatric surgery). Fat around the brachial artery and FMD of the brachial artery was measured among 75 cardiovascular patients.

Results There was good inter-observer and intra-observer reproducibility (coefficient of variance $(\mathrm{CV})<6 \%$ and $<5 \%$ ) and inter-scan repeatability $(\mathrm{CV}<8 \%)$ of the measurement of PVAT. After adjustment for anthropometric indices, demographics and cardiovascular risk factors as appropriate: I) A positive independent association between PVAT and aortic atheroma was detected among obese participants $\left(B M I \geq 30 \mathrm{~kg} / \mathrm{m}^{2}\right)(\mathrm{p}<0.005)$ but not among individuals with intermediate $(\mathrm{BMI}<30$ and $\geq 26)$ and low BMI $(\mathrm{BMI}<26)$. II) Perivascular fat was independently, inversely associated with aortic stiffness among lean patients $(\mathrm{p}<0.0005)$ while the association was independent and positive for obese participants $(p<0.05)$. III) An independent, negative linear correlation between peri-brachial fat and FMD was noted among overweight and obese subjects (BMI $\geq 26)$ $(\mathrm{p}<0.001)$, but not among normal weight participants $(B M I<26)$. IV) PVAT was an independent negative predictor of aortic elasticity among healthy obese individuals $(B M I \geq 30)(p<0.01)$ while it was positively and independently associated with aortic elasticity among lean healthy controls $(\mathrm{BMI} \leq 18)(\mathrm{p}<0.05)$. V) Following weight loss intervention, PVAT reduction was an independent predictor of aortic elasticity improvement in the obese group $(\mathrm{p}<0.05)$.

Conclusions Our results suggest an influence of both generalised and regional excess adiposity on the functional state and the effects of perivascular adipose tissue on dysfunction and remodelling of the underlying vessels.

\section{CARDIOVASCULAR RISK IN ASYMPTOMATIC POTENTIAL SIMULTANEOUS PANCREAS-KIDNEY TRANSPLANT RECIPIENTS IS DETERMINED BY MYOCARDIAL PERFUSION SCINTIGRAPHY}

doi:10.1136/heartjnl-2011-300198.123

V M S Stoll, N S Sabharwal, 00 Ormerod. The John Radcliffe Hospital, Oxford, UK

Introduction More than $50 \%$ of renal transplant recipients will die as a consequence of cardiovascular disease (CVD). Type I diabetics undergoing simultaneous pancreas-kidney transplantation (SPK) are at an even greater risk of CVD. Optimising a patient's cardiovascular status is necessary before SPK transplant surgery. Patients can remain on transplant waiting lists for years. There is little evidence as to how frequently repeat cardiovascular risk assessments are required in asymptomatic patients. Myocardial perfusion scintigraphy is used in SPK patients to detect any asymptomatic myocardial ischaemia or abnormal left ventricular function. This study analyses data from a SPK transplant centre with an annual surveillance programme to aim to establish the suitable frequency of MPS. Methods Potential SPK transplant recipients who had undergone two perfusion scans were included for analysis. An abnormal MPS was defined as either showing a regional wall motion abnormality, inducible ischaemia, or impaired left ventricular function. The scan results were both documented and compared. Angiography results from the study period were also recorded.

Results 99 out of 130 patients on the SPK waiting list in November 2009 had undergone two perfusion scans as part of their 
pre-transplant assessment. The median age was $45 \mathrm{yrs}$ (range 26-63), with $41 \%$ female and a median time between scans of 1.4 yrs (range 0.6-3.0). 59 patients (60\%) had two consecutive normal scans. The remaining 40 patients had at least one abnormal scan. $16 \%$ of patients with a normal 1st scan developed an abnormal 2nd scan within a median period of 1.4 years. 28 (70\%) of the patients with an abnormal MPS underwent angiography, of these 12 required revascularisation (either $\mathrm{PCI}$ or $\mathrm{CABG}$ ). Of the remaining 16 patients; 1 died before angiography and the other 15 patients were treated with medical therapy. Of the 59 patients with two normal scans; 3 underwent angiography during the study period (for new symptoms), 1 of these patients required revascularisation after presenting with an ACS. 2 had minor plaque disease.

Conclusions $40 \%$ of SPK patients on the waiting list have an abnormal MPS. Of the patients with normal scans $5 \%$ required an angiogram because of new symptoms with only $2 \%$ requiring revascularisation. Of the patients undergoing angiography driven by MPS 43\% subsequently underwent revascularisation. The current screening interval is successfully monitoring changes in the patients' cardiovascular status with only one patient requiring an intervention which was not predicted by MPS. Therefore a near annual MPS is a useful, non-invasive means by which to monitor patients at very high risk of asymptomatic cardiovascular disease while awaiting a SPK transplant.

\section{VALIDATION OF THE BCIS-1 MYOCARDIAL JEOPARDY SCORE USING CARDIAC MRI}

doi:10.1136/heartjnl-2011-300198.124

${ }^{1} \mathrm{G}$ D J Morton, ${ }^{2} \mathrm{~K}$ De Silva, ${ }^{1} \mathrm{M}$ Ishida, ${ }^{1} \mathrm{~A}$ Chiribiri, ${ }^{1} \mathrm{~A}$ Indermuhle, ${ }^{1} \mathrm{~A}$ Schuster, ${ }^{2} S$ Redwood, 'E Nagel, 'D Perera. 'King's College London, London, UK; 'uy's and St Thomas' NHS Foundation Trust, London, UK

Introduction The recently described angiographic BCIS-1 Myocardial Jeopardy Score (BCIS JS) was designed to classify the extent of coronary artery disease (CAD). It provides a semi quantitative estimate of the amount of myocardium at risk as a result of severe coronary stenoses $(0=$ no jeopardy; $12=$ maximum jeopardy). Advantages include ease of use and universal applicability including classification of left main stem disease and CABG. However anatomic tests, including the BCIS JS, do not incorporate myocardial ischaemia and scar, which are important for management and prognosis. Cardiac magnetic resonance (CMR) imaging allows reliable assessments of myocardial ischaemia and scar in a single examination and was used to examine the functional relevance of the BCIS JS.

Methods 60 consecutive patients with angina and known or suspected $\mathrm{CAD}$ referred for diagnostic x-ray coronary angiography underwent CMR examination at a single UK centre. CMR included standard functional and scar imaging and also high-resolution $\mathrm{k}-\mathrm{t}$ accelerated adenosine stress and rest perfusion imaging at 1.5T (40 patients) or 3T (20 patients). Expert observers blinded to the clinical data analysed the angiographic and CMR data. The BCIS JS was calculated from visual analysis of the coronary angiogram. CMR perfusion and scar data were segmented according to the standard 17-segment model excluding the apex. Segments were subdivided into equal endo- and epicardial sub-segments, each assigned $3 \%$ of the total myocardial volume and classified as normal, ischaemia or scar. Myocardial ischaemia and scar burden were calculated and correlated with the BCIS JS individually and as a combined score (Abstract 124 figure 1).

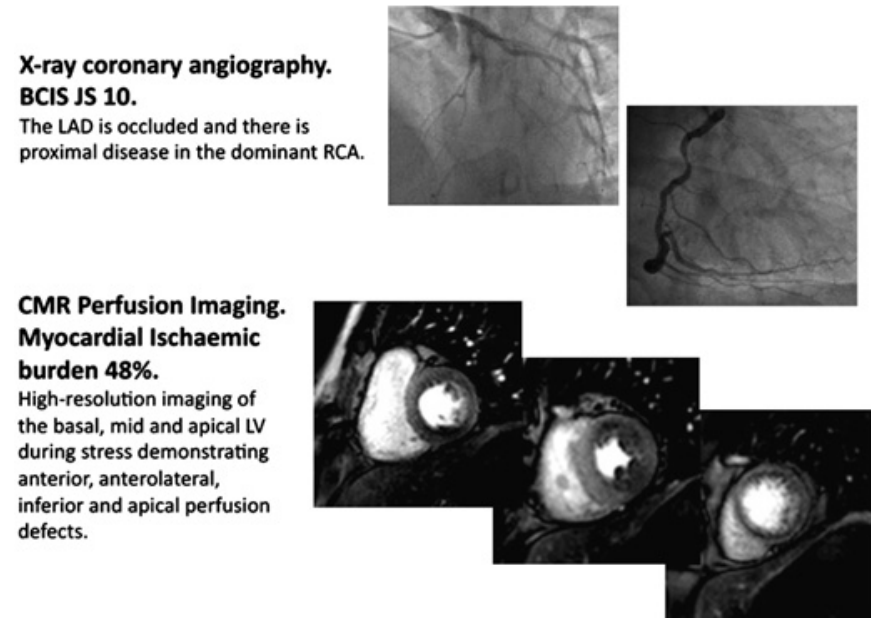

Abstract 124 Figure 1

Results Patient characteristics are summarised in the Abstract 124 table 1.2 patients were excluded (1 claustrophobia; 1 incomplete imaging data). Mean interval \pm SD between CMR and coronary angiography was $40 \pm 47$ days. 13 patients (22\%) with no history of myocardial infarction had CMR evidence of prior infarction. There was a strong correlation between the BCIS JS and myocardial ischaemic burden: Pearson's $r=0.75, p<0.00001$ (Abstract 124 figure 2 ). The BCIS JS was also correlated with the combined burden of scar and ischaemia: $r=0.77, p<0.00001$. There was no difference between 3T and 1.5T CMR imaging. Area under the receiver-operating characteristic curve for BCIS JS to detect $\geq 10 \%$ myocardial ischaemic burden was 0.87 (95\% CI 0.77 to 0.97 ). BCIS JS $\geq 6$ predicted $\geq 10 \%$ myocardial ischaemic burden with sensitivity $68 \%$ and specificity $90 \%$.

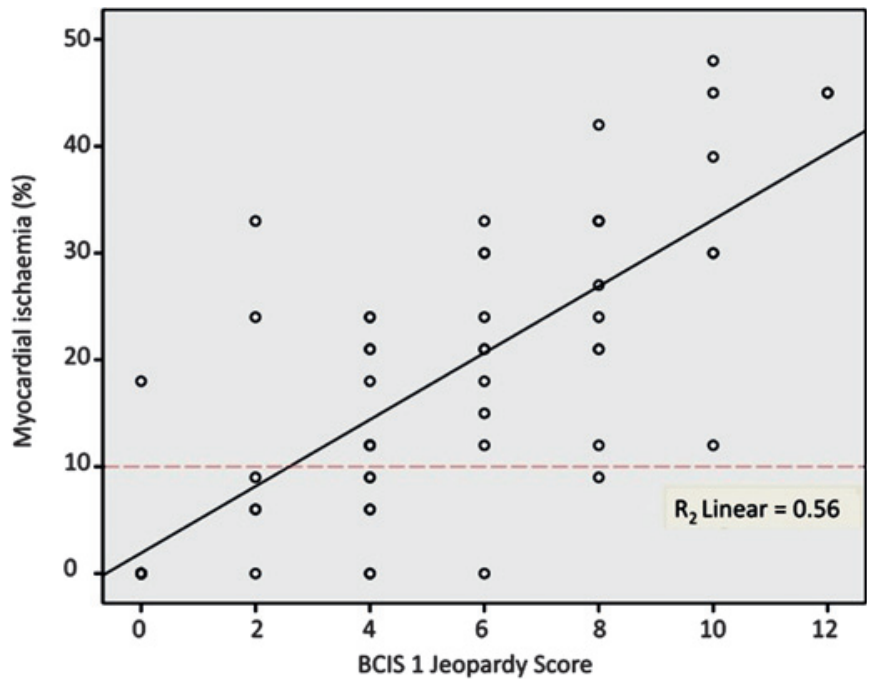

Abstract 124 Figure 2 Correlation between myocardial ischemic burden and BCIS JS.

Conclusions The BCIS JS correlated well with ischaemic burden on CMR. A BCIS JS $\geq 6$ predicts the prognostically important ischaemic threshold of $10 \%$ with high specificity. As expected, the correlation is imperfect which is likely to be a result of difficulty predicting haemodynamic effects of angiographically moderate disease, microvascular disease and limitations of CMR imaging. 